\title{
EMOTIONAL AND FAMILIAL DETERMINANTS OF ELEVATED BLOOD PRESSURE IN BLACK AND WHITE ADOLESCENT FEMALES*
}

\author{
ERnest H. Johnson, $\dagger$ Nicholas J. SCHORK $\dagger$ and Charles D. SPIELBERGER $\ddagger$
}

(Received 5 March 1987; accepted in revised form 21 May 1987)

\begin{abstract}
The relationship between various measures of the expression and experience of anger (and anxiety), traditional risk factors and blood pressure were examined in a sample of black and white female adolescents who were enrolled in health science courses in Tampa, Florida. Whereas a number of the personality and traditional risk factors were significantly correlated with blood pressure, body mass $\left(w / h^{2}\right)$ was the most consistent predictor of blood pressure (SBP and DBP) for both black and white females. The findings examining the relationship between suppressed anger (AX/Anger-In) and blood pressure show that SBP and DBP was significantly higher for both black and white females who frequently harbored grudges and held in their angry feelings. In addition, the relationship of AX/Anger-In scores and blood pressure appears to have a 'threshold'.
\end{abstract}

\section{INTRODUCTION}

THE ASSOCIATION between suppressed anger and elevated blood pressure has received a considerable amount of research attention over the past 40 years [1-5]. The majority of studies, however, have included only young white males as subjects, and few investigations have examined the association between emotional factors and blood pressure within and between both black and white subpopulations. Early clinical observations and interviews with hypertensive patients $[1,6-8]$ have provided a great deal of the stimulus for recent investigations. In fact, the so called 'specificity hypothesis' that was proposed by Franz Alexander has been the focus of the most recent studies. Alexander [1] proposed that the chronic elevation of blood pressure among persons with hypertension was maintained by the frequent experience of angry feelings that were suppressed. The hypertensive patient was considered to be in an 'emotional paralysis' where he was unable to freely express angry feelings or aggressive behaviors because of extremely strong competing tendencies and traits toward passive, dependent, anxious, submissive and fearful behaviors [9-12].

Results from the few investigations of female subjects [9-12] have shown a consistent relationship between elevated blood pressure and various emotional factors. For example, Harris et al [9] had observers rate a sample of borderline hypertensive female undergraduates using an Adjective Check List as they engaged in emotionally provoking psychodramas. The borderline hypertensives were described as hotheaded, rash, angry, daring, hurried and tense. In a followup of the same subjects

*This work was supported in part by a new investigator research grant to Dr Johnson from the National Institutes of Health (HL33414-01A1) and in parts from funds to Dr Spielberger from R. J. Reynolds, Inc. Address all correspondence to Dr Ernest H. Johnson.

$\nmid$ Division of Hypertension, Department of Internal Medicine, 3918 Taubman Center, The University of Michigan Medical Center, Ann Arbor, 48109-0356, U.S.A.

$\ddagger$ Center for Research in Behavioral Medicine and Health Psychology, University of South Florida, 4202 Fuwler Avenue, Tampa, FL 33620, U.S.A. 
as college seniors [11], extensive interviews were conducted in the presence of an observer who classified each subject as either borderline hypertensive or normotensive based on observations made during the interview and responses to an Adjective Check List. Borderline hypertensives were described as being more hostile, excitable, egocentric, moody, nervous, anxious and angry than the normotensives. Kalis et al. [11] concluded that these young borderline hypertensive women were unable to appropriately express their anger and hostility, which resulted in an increase in anxiety, muscle tension, and overall emotional reactivity.

Kalis et al. [10] attempted to cross-validate the findings of Harris et al. [9] with a sample of 14 females diagnosed as being essential hypertensives. Subjects were engaged in a psychodrama which required a friendly, cooperative behavior. Observers rated the essential hypertensives as hostile, aggressive, self-punitive, emotionally guarded, nervous resentful and inappropriate in their control over anger and hostility. A more recent study, using objective self-report measures of anger, to examine the relationship between emotional factors and cardiovascular risk factors among adolescents was reported by Siegel [12]. Regression analyses, controlling for age and sex, showed that elevations in both systolic and diastolic blood pressure were associated with higher scores on the 'anger-out' measure. However, adding the sex-by-anger interaction to the equation revealed that the relationship between anger-out and blood pressure was accounted for primarily by the male adolescents. It should be noted that the sample of black adolescents was too small to conduct analyses by race.

The relationship between anger coping types and diastolic blood pressure (DBP) was examined among adult black and white female subjects from the Detroit study $[13,14]$. The women in this study were classified in terms of their habitual anger-coping responses to hypothetical anger-provoking situations which required either an 'anger-in' or 'anger-out' response to an unjustifiable provocation by an authority figure (boss, policeman) or a family member (spouse). Results indicated that an anger-in coping response was associated with higher DBP for both black and white females. The relationship between anger expression and DBP was, however, mediated by the degree of socioenvironmental stress. In other words, the relationship between DBP and anger-in (suppressed anger) was observed only for black females who resided in high stress neighborhoods and white females who resided in low stress areas. In a more recent study, the relationship between blood pressure and various psychosocial factors was examined in a probability sample of male and female adults who resided in the state of Michigan [5]. Among both women and men, those who generally do not express their emotions (including anger) and who harbor grudges and aggressive impulses had higher diastolic blood pressure. This relationship was reported to be independent of the major risk factors for heart disease such as age, weight, alcohol consumption, smoking, and diagnosis of hypertension.

Although the evidence supporting a causal association between emotional factors and blood pressure is at best weak, further research is warranted for a number of reasons. First, a variety of methodological problems have made it difficult to directly link suppressed anger and other emotional factors with elevated blood pressure [2, 4]. Most of the data are cross-sectional and simply compare hypertensive and normotensives on self-report measures of anger. Furthermore, some of the studies 
comparing hypertensives and normotensives on psychological measures are biased because the psychological findings may be influenced by the 'diagnosis' of hypertension. In other words, the findings are subject to questions as to whether they relate to the mechanisms of elevated blood pressure or whether the emotional experiences are a secondary consequence of the long-standing diagnosis of hypertension. Second, the measures of suppressed anger and other emotional factors vary dramatically across studies and are largely psychometrically inadequate (i.e. no evidence of the reliability and/or validity of the measures). Finally, past studies are limited because of the failure to include measures of both the experience and expression of anger and don't typically use measures that distinguish between the intensity of anger (and anxiety) as an emotional state from individual differences in anger (and anxiety) proneness as an enduring personality trait $[3,4]$. By using empirically derived psychologic measures and adolescent subjects who have never been diagnosed as having hypertension, the present inquiry was able to address some of the limitations of previous research.

The present study examines whether the associations between blood pressure and various dimensions of the expression (Anger-In, Anger-Out) and experience (intensity, frequency) of anger (and anxiety), as well as traditional risk factors, differ between black and white female adolescents. To our knowledge, this is the first study to examine the relationship between blood pressure and emotional factors for both black and white adolescent females. The rationale for testing for these relationships is derived from our previous research with adolescent males [4] which showed that those black and white males who generally harbored grudges and frequently suppressed their anger had higher systolic blood pressure; diastolic blood pressure was higher only for the white males who frequently held in their angry feelings. In addition, familial factors, weight and excessive salt usage, were found to be independent predictors of systolic and diastolic blood pressure only for the white adolescent males. The previous research with adolescent males showed that the relationship between Anger-In scores and blood pressure appeared to have a 'threshold', with the risk of elevated SBP and DBP being relatively low for Anger-In scores up to 18 , though it increases rapidly for both black and white males above this score. The present investigation will determine if similar associations between Anger-In scores and blood pressure are obscrved for adolescent females.

\section{METHODS}

\section{Subjects}

The participants for this study were 171 black and 279 white female high school sophomores ranging between 15 and $17 \mathrm{yr}$ of age who had not been previously diagnosed as hypertensive. All subjects were enrolled in health science courses at seven of the nine high schools in and around Tampa (Hillsborough County), Florida, during the 1981-82 school year. Blood pressure and personality data were obtained from approximately $95 \%$ of the students who were present in the classrooms during the days of the study. The non-participants consisted primarily of those students who 'forgot' to obtain their parents' permission to participate in this phase of the investigation.

\section{Measurement instruments}

The following self-report instruments were employed in the study, and the individual items and response formats comprising each scale can be obtained from the authors.

(a) State-Trait Personality Inventory (STPI). The STPI was developed by Spielberger et al. $[15]$ to measure state and trait anger, anxiety and curiosity. The STPI State Scale consists of three ten-item 
subscales for measuring the intensity of anger (State Anger), anxiety (State Anxiety) and curiosity (State Curiosity), while the Trait Scale measures the general disposition to experience frequent feelings of anger (Trait Anger), anxiety (Trait Anxiety) and curiosity (Trait Curiosity). The Trait Anger scale also has two subscales. The first is referred to as the Trait Anger/Temperment Scale and it assesses the general disposition to experience anger while the Trait Anger/Reaction scale measures the disposition to experience intense anger when provoked. The directions for the State Scales were altered so that it was possible to determine the intensity of emotions at the time the subjects' blood pressures were measured. The high internal consistency reliability of the STPI T-Anger scales are reflected in the relatively high alpha coefficients $(r=0.82-0.85)$ and large item remainder correlations $(0.42-0.73)$ that were found in a factor analysis of the scales using college students and Navy recruits.

(b) Anger Expression Scale $(A X)$. The AX was developed by Spielberger et al. [16] and Johnson [17] to measure the frequency that anger is expressed. The AX yields a total scale score, AX/Anger-In (suppressed anger) and AX/Anger-Out subscale scores. The internal consistency of the AX total scale and the Anger-In and Anger-Out subscales, as measured by Cronbach's alpha coefficients, ranged from 0.70 to 0.84 for the subjects in the present investigation. Similar findings were reported for 911 undergraduate students ( 335 males, 576 females) by Pollans [18]. Item remainder correlations for the Anger-In and Anger-Out subscales ranged from 0.30 to 0.63 and the test-retest correlations for the AX and its subscales, computed for 107 subjects ( 32 males, 75 females) over an 8-10-week time interval, ranged from 0.58 to 0.75 . A more thorough description of psychometric properties of the $\mathrm{AX}$ has been described elsewhere [16-18].

(c) Smoking Behavior Questionnaire ( $S B Q)$. The SBQ is a 50-item self-report questionnaire developed to elicit specific information about the cigarette smoking habits of students and their family members. In the present study, only 17 of the items were administered and data analysis will be presented only for the percentage of cigarette smokers and nonsmokers. The psychometric properties of the SBQ have been described elsewhere by Spielberger et al. [19].

(d) Harburg Anger-In/Anger-Out Scale (HAX). Modeled after a scale developed by Harburg et al. $[13,14]$ the HAX was used in the present study to assess the degree that anger is expressed outwardly. In order to measure anger expression, hypothetical situations that required subjects to report how they would express their feelings (Anger-In or Anger-Out) if they were treated unjustly by a teacher, a movie patron and a club president. The subjects also rate themselves on a four-point scale according to how much guilt they would experience if they became angry at the person responsible for the injustice in the vignette. The HAX items are scored to create an anger expression (Anger-Out) index using the procedures reported by gentry et al. [20].

(e) State Anger Reaction Scale ( $S$-Anger/RX). The S-Anger/RX was developed to measure the intensity of anger experienced in stressful and frustrating social situations [17]. The S-Anger/RX yields a total scale score, and two subscales for angry responses to time-pressure (TP) and evaluative-threatening (ET) situations. The internal consistency of the S-Anger/RX total scale and its time pressure (TP) and evaluative-threatening (ET) subscales as measured by alpha coefficients ranged from 0.81 to 0.87 for the subjects in the present study. Item remainder correlations for the TP and ET subscales ranged from 0.42 to 0.67 . A more thorough description of psychometric propertics of the S-Angcr/RX scale has been described elsewhere $[17,18]$.

(f) Dietary-Checklist. This instrument measures the frequency that foods rich in sodium, sugar and cholesterol are eaten. It consists of 52 food items and requires the subjects to rate how often they use these foods on a four-point scale. Some of the items in the check list were taken from a Kitchen Inventory of Salty Junk Foods developed by Karp, Williams and Grout [21]. Additional items were added based on a nurse's evaluation of the contents of vending machines located on or near the high school campuses.

(g) Family History of Heart Disease Questionnaire. This is a 20-item questionnaire to determine the history of hypertension and heart disease among the natural parents and grandparents of the subjects for the present study. The responses to this questionnaire were obtained from the parents at the same time that they completed the consent statement for their child to participate in the investigation.

\section{PROCEDURE}

The complete details of the sample selection and data acquisition are provided elsewhere $[4,16,17]$. In brief, the data was obtained from students enrolled in Health Sciences courses during regular class periods focused on diseases of the circulatory system. All students were given a test booklet containing the instructions and questionnaires. The students were informed that the meaning of their measurements would be explained during a 'Hypertension Study Unit' that was presented by members of the research team at the next meeting of their class. Shortly after the students began work on the questionnaires, the first students were escorted to the back of the classroom where research assistants obtained the 
following measurements: (1) height and weight; (2) blood pressure; and (3) self-reports of the feelings experienced while blood pressure was being taken (STPI State Scale). Height and weight were recorded by the first research assistant with the standard instruments used by school personnel, while blood pressure was measured by the second assistant using an automatic blood pressure monitor that is manufactured by the Sears Company (Model No. 8-2153), which has a microphone located within the cuff to pick up Korotkoff sounds. The cuff was attached to the right forearm, above the elbow and just under the bicep muscle, with the microphone positioned over the brachial artery. The blood pressure monitor was programmed such that Korotkoff sounds first appeared as systolic blood pressure and then diminished sharply in amplitude before disappearing. The point of the disappearance of sound rather than the point of 'muffling' was used as an estimate of the diastolic blood pressure. Immediately after the cuff was deflated systolic blood pressure (SBP), diastolic blood presure (DBP) and pulse rate were displayed on a digital readout and recorded by the research assistant. The students were kept unaware of their blood pressure levels until they completed the study and departed from the classroom. The principal investigator (EHJ) met individually with each student who had elevated blood pressure (SBP $\geq 135$ /or $\mathrm{DBP} \geq 85 \mathrm{mmHg}$ ) to schedule a follow-up examination. After height and weight were measured, the first blood pressure measurement was obtained after each student was seated in an unpadded chair for approximately 2 to $5 \mathrm{~min}$. A second blood pressure was taken about $30 \mathrm{sec}$ after the first measurement. Immediately following the blood pressure measures, the students were administered the STPI State Scales by the third research assistant with instructions to report how they felt at the time the blood pressure was being taken. After completing the STPI State Scales, the student was escorted back to her seat, and instructed to continue working on the questionnaires in the test booklet.

\section{RESULTS}

In the present study the average systolic and diastolic blood pressure measures were used in all analyses because of the high Pearson Product-moment correlations between the first, second and average blood pressure measurcs for the black and white females. The correlation between the systolic blood pressure measures ranged from 0.68 to 0.98 , while the correlations between diastolic blood pressure ranged from 0.86 to 0.98 . Black and white females were treated separately in all analyses because the results of previous analyses [17] showed that the average SBP (black $=121 \mathrm{mmHg}$; white $=112 \mathrm{mmHg}$ ), DBP (black $=67 \mathrm{mmHg}$; white $63=\mathrm{mmHg}$ ) and distribution of blood pressure (systolic and diastolic) were significantly different ( $p<0.001$ for all comparisons) for the two groups.

Table I presents the correlations between the personality measures, traditional risk factors and both SBP and DBP stratified by race. For black and white females, those who frequently suppress their anger - as measured by the AX-Total, AX/ Anger-In, or Harburg Anger In/Out - have higher SBP. Whereas similar findings were also revealed for DBP among black females, neither measure of anger expression correlated significantly with DBP for white females. The significant correlations between AX/Anger-Out scores and both SBP and DBP for black females indicates that blood pressures were higher for those black females who refrain from expressing their anger outwardly at people or objects in their environment. Among black females, those who reported experiencing intense anxiety (State Anxiety) reactions while their blood pressures were being measured have higher SBP and DBP. Those black females who reported experiencing intense angry reactions (S-Anger/RX) in Time Pressured (TP) situations, as well as intense anger (State Anger) reactions while their blood pressures were being measured had higher SBP and DBP. Although neither of the S-Anger/RX measures correlated significantly with SBP for white females, those who reported higher scores on all three of the S-Anger/RX scales had higher DBP. Black females who reported having a strong disposition to experience intense anger when provoked (Trait Anger/Reaction) had higher DBP. 
TABLE I. -CORRELATIONS BETWEEN PERSONALITY, TRADITIONAL RISK FACTORS, AND BLOOD PRESSURE BY RACE FOR ADOLESCENT FEMALES

\begin{tabular}{|c|c|c|c|c|}
\hline \multirow[b]{2}{*}{ Personality measures } & \multicolumn{2}{|c|}{ Black $(n=171)$} & \multicolumn{2}{|c|}{ White $(n=279)$} \\
\hline & systolic & diastolic & systolic & diastolio \\
\hline Trait Anxiety & -0.07 & -0.02 & -0.16 & -0.04 \\
\hline State Anxiety & $0.22 *$ & $0.20^{*}$ & 0.16 & 0.13 \\
\hline Trait Anger & 0.01 & 0.16 & -0.12 & 0.06 \\
\hline$T \cdot$ Anger $/ T$ & -0.07 & 0.05 & 0.17 & 0.02 \\
\hline T-Anger/R & 0.06 & $0.25^{*}$ & -0.03 & 0.11 \\
\hline State Anger & 0.14 & $0.20^{*}$ & 0.05 & -0.05 \\
\hline S-Anger/RX & 0.14 & 0.11 & 0.11 & $0.24 *$ \\
\hline TP-Anger & $0.17+$ & $0.22 *$ & 0.13 & $0.21^{*}$ \\
\hline ET-Anger & 0.05 & -0.05 & 0.06 & $0.21 *$ \\
\hline AX-Total & $-0.30^{*}$ & $-0.28^{*}$ & $-0.29^{*}$ & -0.10 \\
\hline Anger-In & $0.27 *$ & $0.22^{*}$ & $0.24 *$ & 0.10 \\
\hline Anger-Out & $-0.20^{*}$ & $-0.17 \dagger$ & -0.12 & -0.01 \\
\hline Harburg Anger-In/Out & $-0.24^{*}$ & $-0.26^{*}$ & $-0.30^{*}$ & -0.11 \\
\hline Guilt & 0.07 & -0.02 & -0.05 & -0.03 \\
\hline \multicolumn{5}{|l|}{ Traditional Risk Factors } \\
\hline MFHBP & $0.18 \dagger$ & $0.18+$ & $0.26^{*}$ & $0.20^{*}$ \\
\hline FFHBP & -0.05 & $0.22 *$ & 0.08 & 0.15 \\
\hline MFHD & -0.09 & 0.08 & $0.33^{*}$ & $0.20^{*}$ \\
\hline FFHD & -0.08 & 0.05 & $0.23^{*}$ & 0.14 \\
\hline Quetelet Index $\left(w / h^{2}\right)$ & $0.26^{*}$ & $0.30 *$ & $0.30^{*}$ & $0.25^{*}$ \\
\hline Salt & $0.17 \dagger$ & $0.28^{*}$ & 0.16 & 0.15 \\
\hline Smoking & 0.01 & 0.04 & 0.10 & 0.14 \\
\hline
\end{tabular}

$*_{p}<0.001$.

$+p<0.01$.

$\ddagger$ Mother’s family history of hypertension.

$\S$ Father's family history of hypertension.

"Mother's family history of heart disease.

ๆFather's family history of heart disease.

The bottom portion of Table I shows the correlations between the traditional risk factors and blood pressure for the adolescent females. For black and white females, those who have a parental history of hypertension (mother only) have higher SBP and DBP; the father's family history of hypertension was significantly correlated only with DBP among the black females. Among the white females, those who have a parental history of heart disease (mother only) have higher SBP and DBP; the father's family history of heart disease was significantly correlated only with SBP for the white females. For black and white females, those who have a greater body mass $\left(w / h^{2}\right)$ have higher SBP and DBP. Among black females, excessive salt usage shows a significant positive association with both SBP and DBP.

To identify from among the significant personality and traditional risk factor measures the independent predictors of blood pressure, stepwise multiple regression was employed. The minimum $F$ of variables to be entered into the linear equation was specified at $p<0.01$. A forward stepwise algorithm then selected those personality and risk factor measures which explained a significant proportion of the variance in blood pressure not explained by the other variables in the model. Table II presents the findings for these analyses. Among the white females, the results of this analysis indicated that five variables (MFHD, Quctclet Index, IIarburg Anger 
TABLE II. - SUMMARY OF THE REGRESSION ANALYSES USING PERSONALITY AND RISK FACTORS TO PREDICT BLOOD PRESSURE FOR ADOLESCENT FEMALES

\begin{tabular}{|c|c|c|c|}
\hline \multicolumn{2}{|c|}{ Black $(n=171)$} & \multicolumn{2}{|c|}{ White $(n=279)$} \\
\hline variable & SBP & variable & SBP \\
\hline $\begin{array}{c}\text { AX-Total } \\
w / h^{2}\end{array}$ & $\begin{array}{l}0.09(-0.30)^{*} \\
0.14(0.23)\end{array}$ & $\begin{array}{c}\text { MFHD } \\
w / h^{2} \\
\text { Harburg In/Out } \\
\text { AX/Anger-In } \\
\text { T-Anxiety }\end{array}$ & $\begin{array}{lr}0.11 & (0.33) \\
0.18 & (0.29) \\
0.25 & (-0.29) \\
0.27 & (0.18) \\
0.32 & (-0.24)\end{array}$ \\
\hline variable & DBP & variable & DBP \\
\hline $\begin{array}{c}w / h^{2} \\
\text { T.Anger } / \mathrm{R} \\
\text { Salt }\end{array}$ & $\begin{array}{ll}0.09 & (0.30) \\
0.15 & (0.27) \\
0.20 & (0.23)\end{array}$ & $\begin{array}{c}w / h^{2} \\
\text { S-Anger/RX } \\
\text { MFHBP }\end{array}$ & $\begin{array}{l}(0.25) \\
(0.25) \\
(0.22)\end{array}$ \\
\hline
\end{tabular}

*Partial correlation adjusted for all other variables in the final step of the regression analyses are presented within parentheses.

Expression, AX/Anger-In, and Trait-Anxiety) accounted for $32 \%$ of the total SBP variance. The MFHD variable, which entered first into the equation, correlated 0.33 with SBP and accounted for approximately $34 \%$ of the variance explained by the five variables in the regression model. The results of the stepwise regression analysis for DBP indicated that three variables (Quetelet Index, S-Anger/RX, and MFHBP) accounted for $16 \%$ of the total DBP variance for white females. The body mass index $\left(w / h^{2}\right)$ was the first variable to enter the regression equation and it accounted for approximately $38 \%$ of the variance explained by the three variables in the regression model. Among the black females, the results of the regression analysis predicting SBP indicated that two variables (Anger Expression-Total Scale and Quetelet Index) accounted for $14 \%$ of the total variance while the findings for DBP indicated that three variables (Quetelet Index, Trait Anger/Reactions, and Salt Usage) accounted for $20 \%$ of the total DBP variance. The correlation of 0.09 between SBP and AX-Total indicatcd that anger expression alone accounted for approximately $64 \%$ of the variance while body mass accounted for approximately $45 \%$ of the DBP variance explained by the three variables in the regression model.

In an effort to clarify the nature of the relationship between the frequency that angry feelings are held in (AX/Anger-In) and blood pressure, the female adolescents were divided into five subgroups on the basis of their AX/Anger-In scores. Since the number of white females exceeded the number of black females, and it was considered desirable to use the same cut-off scores for both ethnic groups, the white females were divided into five subgroups using an unbiased 'counting off' procedure, in which the extreme groups were as nearly equal in size as possible. The same cut-off scores were then used in defining comparable Anger-In subgroups for the black females. The Anger-In cut-off scores for the five subgroups of white females, and the number of students in each of these groups were: 8-13 $(n=55)$, 14-15 $(n=58), 16-18(n=81), 19-22(n=49), 23-32(n=36)$. Using the same cut-off scores for the black females, the number of students in the five Anger-In 


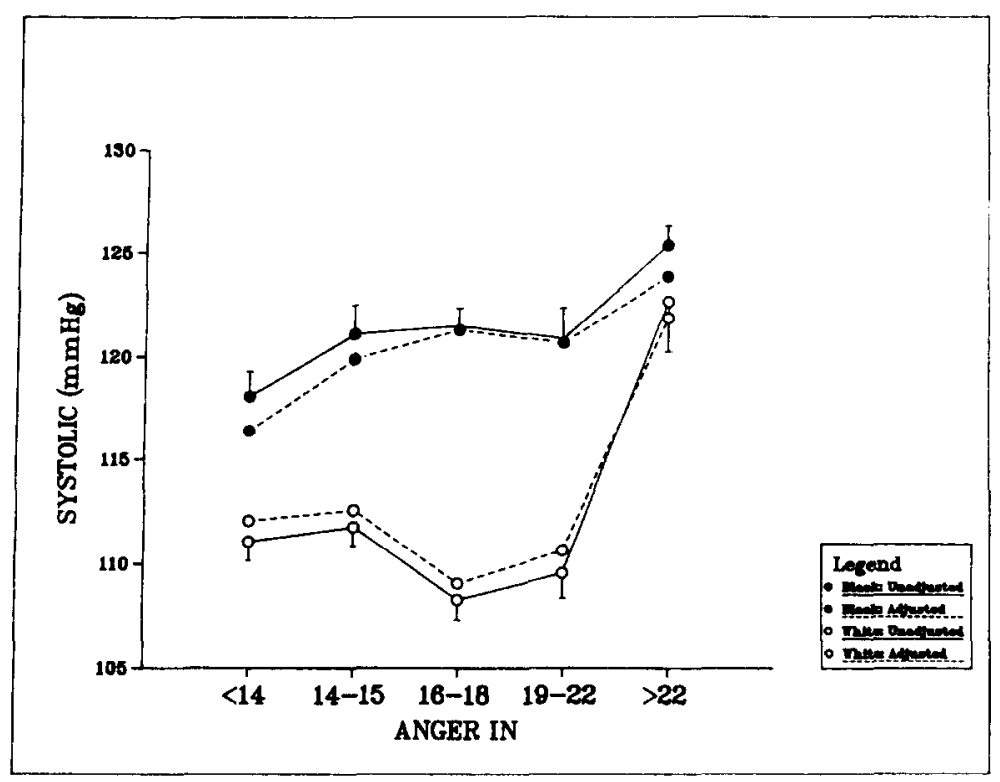

FIG. 1. - Mean systolic blood pressure ( \pm SEM) for five groups of black and white female adolescents defined on the basis of increasing scores on the Anger-In subscale.

subgroups were $29,18,43,29$ and 52 , respectively. Figure 1 reports mean SBP in millimeters of mercury $(\mathrm{mmHg})$ for five subgroups of white and black female students defined as a function of increasing AX/Anger-In scores. The standard error of the mean (SEM) for the SBP values are also platted.

Differences in SBP for white and black females in the five Anger-In groups were evaluated by a 2 factor analysis of covariance in which Anger-In and race were the independent variables and the traditional risk factor measures were controlled. Although both unadjusted and adjusted blood pressure values are presented, only the analyses for the adjusted values are given. This was essentially due to the lack of a significant difference between the unadjusted and adjusted blood pressure values. In this analysis, the main effects of Anger-In $[F(4,440)=8.90, P<0.001]$ and race $[F(1,440)=29.68, P<0.01]$, and the Anger-In by race interaction $[F(4,440)=2.99, P<0.001]$ were statistically significant. The large Anger-In effect reflected the finding that SBP for both black and white females who had the highest Anger-In scores was substantially higher than for females who were low on Anger-In. Whereas the race main effect indicated that the SBP for black females was consistently higher than for the white females, the Anger-In by race interaction show that the SBP for the black females began to increase at a lower level of Anger-In than for the white females and was approximately $6-11 \mathrm{mmHg}$ higher for the first four Anger-In groups. A similar procedure was also used to evaluate differences in DBP as a function of Anger-In and race. The results of this analysis show that the main effects of Anger-In $[F(4,440)=3.45, P<0.01]$ and race $[F(1,440)=3.57, P<0.05]$ were highly significant. However, the Anger-In by race interaction was not significant $[F=0.77]$ in this analysis. These results indi- 
cated that the DBP for the black and white females who had the highest Anger-In scores was substantially higher than for both groups of females who had low AngerIn scores, as can be seen in Fig. 2. The race main effect reflected the finding that DBP for black females was uniformly higher than for the white females at the levels of the Anger-In variable.

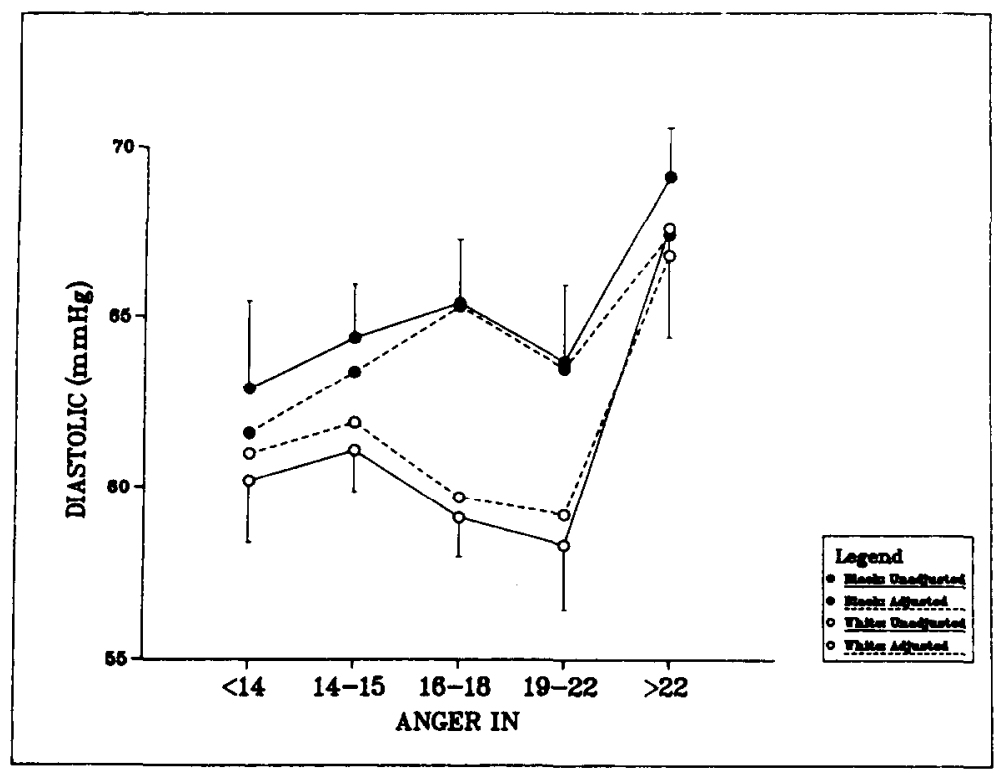

FIG. 2. - Mean diastolic blood pressure ( \pm SEM) for five groups of black and white female adolescents defined on the basis of increasing scores on the Anger-In subscale.

\section{DISCUSSION}

It has been demonstrated here that certain emotional factors are related to blood pressure among both black and white adolescent females. Although there is a large body of literature indicating that anger conflict is related to blood pressure, there appears to be no general agreement regarding the dimensions of anger that are related to hypertension for either blacks or whites. For example, the present investigation revealed some subtle differences in the association between the various dimensions of anger and blood pressure for black and white adolescent females. Anger expression, as measured by the AX-Total scale, was significantly related to both SBP and DBP among black females, while two comparable measures of anger expression (AX/Anger-In; Harburg In/Out) remained significantly related only to SBP among white females. Whereas neither of these measures of anger expression was significantly related to DBP for white females, a measure of intense angry reactions to unjust social situations (S-Anger/RX) remained significantly related to DBP after adjusting for the risk factor measures. It should also be noted that a similarly conceived measure of the disposition to experience intense anger when 
provoked (Trait Anger/Reaction) was a significant predictor of DBP for black females.

The present investigation replicates some aspects of previous findings [9-13] regarding anger and blood pressure. However, it is extremely difficult to compare findings from this investigation with others because suppressed anger was measured with a self-report questionnaire designed to assess the frequency that angry feelings are suppressed rather than a coping response to hypothetical $[13,14]$ anger-provoking situations. It is equally more difficult to consider the diverse implications of the conclusions regarding what the association between anger conflict and blood pressure means because the association is not consistent for both SBP and DBP. Unfortunately, many investigations of the relationship between blood pressure and emotional factors report on only SBP or DBP which makes it difficult to speculate about underlying mechanisms such as increased cardiac output or increased peripheral resistance.

Another important finding of the present inquiry was the relative consistency of the relationship between blood pressure and body mass for both black and white females. Although the relationship between blood pressure and body weight has been studied less extensively in adolescents, the findings from the present investigation are in agreement with previous studies [22]. We considered whether suppressed anger was related to body mass and other risk factors that were related to blood pressure. Could it be that female adolescents who are more obese, smoke, or who consume more salty junk foods are more likely to frequently harbor grudges and that suppressed anger is not independently related to blood pressure? The findings of an analysis of covariance examining the relationship between blood pressure and the frequency that angry feelings are suppressed suggests that this is not the case. On the whole, the relationship between Anger-In and blood pressure held up after controlling for body mass, salt usage, smoking, and family history factors. There is, however, the possibility that controlling for certain indicators of psychosocial stress and social background (e.g. parental education/income levels, employment/marital status, distressful life events) or behavioral factors (e.g. exercise habits, alcohol usage) that were not available for study could modify the associations between blood pressure and suppressed anger. In future studies, the role of these factors in mediating the relationship between elevated blood pressure and personality factors should be examined.

In conclusion, the data are supportive of an association between anger conflict and blood pressure for both black and white adolescent females, although it should be noted that the anger measures accounted for a small percentage of the variance in blood pressure. Because of the cross-sectional nature of our data, definitive statements cannot be made as to whether anger precedes or is a consequence of elevated blood pressure. However, these data are not biased by the possibility that anger may be a reaction to a diagnosis of hypertension, as has been a major problem with much of the cross-sectioned data from studies of adults. To better understand the etiologic significance of anger in hypertension, it would appear necessary that future research efforts be directed at identifying the pathophysiologic and hemodynamic abnormalities underlying the suppressed anger coping style. It is conceivable that the pathophysiologic and hemodynamic profile of black and white 
hypertensives who frequently harbor grudges and have conflicts about expressing their anger are markedly different.

\section{RFFFRFNCES}

1. AlexAnder FG. Emotional factors in essential hypertension: presentation of a tentative hypothesis. Psychosomatic Med 1939; 1: 175-179.

2. DIAMOND EL. The role of anger and hostility in essential hypertension and coronary heart disease. Psychol Bull 1982; 92: 410-433.

3. Schneider R, Egan B, Johnson EH, Drobney H, Julius S. Anger and anxiety in borderline hypertension. Psychosomatic Med 1986; 48: 242-248.

4. JOHNSON EH, SPIELBERGER CD, WORDEN TJ, JACOBS GA. Emotional and familial determinants of elevated blood pressure in black and white adolescent males. J Psychosomatic Res 1987; 31: $287-300$.

5. Cottington EM, Rrock BM, Housf IS, Hawthornf. VM. Psychosocial factors and blond pressure in the Michigan statewide blood pressure survey. Am J Epidemiol 1985; 121: 515-529.

6. Binger CAL, ACKerman NW, Cohn AE. Personality in arterial hypertension. Psychosomatic Med Monographs, 1945. New York: American Society for Research in Psychomatic Problems.

7. SaUl L. Hostility in cases of essential hypertension. Psychomatic Med 1939; 1: 153-161.

8. VANDERVALK JM. Blood pressure changes under emotional influences in patients with hypertension and control subjects. J Psychosomatic Res 1957; 2: 134-146.

9. Harris RE, Sokalow M, Carpenter LG, Freedman D, Hunt SP. Response to psychological stress in persons who are potentially hypertensive. Circulation 1953; 7: 874-879.

10. Kalis BL, Harris RE, BenNett CF, Sokalow M. Personality and life history factors in persons who are potentially hypertensive. J Nervous \& Mental Dis 1961; 132: 457-468.

11. Kai is BL, Harris RE, Sokalow M, Carpenter LG. Response to psychological stress in patients with essential hypertension. Am Heart J 1957; 53: 572-578.

12. Siegel JM. Anger and cardiovascular risk in adolescents. Health Psychol 1984; 3: 293-313.

13. HaRburg E, Blakelock EH, RoePER PJ. Resentful and reflective coping with arbitrary authority and blood pressure: Detroit. Psychosomatic Med 1979; 3: 189-202.

14. HARBURG E, HAVENSTEIN L. Parity and blood pressure among four race-stress groups of females in Detroit. Am J Epidemiol 1980; 3: 356-366.

15. SPIElBerger CD, JACOBS GA, BAKER L, et al. Preliminary manual for the state-trait personality inventory (STPI). Center for Research in Behavioral Medicine and Health Psychology 1979; University of South Florida, Tampa.

16. Spielberger CD, Johnson EH, Russell SF, et al. The experience and expression of anger: construction and validation of an anger expression scale. In Anger and Hostility in Cardiovascular and Behavioral Disorders (Edited by CHESNEY MA, RosenMAN RH). New York: McGraw-Hill, 1985.

17. JoHNSON EH. Anger and anxiety as determinants of elevated blood pressure in adolescents: the Tampa study. Doctoral dissertation, 1984; University of South Florida, Tampa.

18. Pollans $\mathrm{CH}$. The psychometric properties and factor structure of the anger expression scale. Master's Thesis, 1983; University of South Florida, Tampa.

19. Spielberger CD, Jacobs GA, Crane R, Russell SF. On the relation between family smoking habits and smoking behavior of college students. Int Rev appl Psy 1983; 31: 53-69.

20. Gentry WD, Chesney AP, Gary HG, Hall RP, Harburg E. Habitual anger coping styles: I. Effect on mean blood pressure and risk for essential hypertension. Psychosom Med 1982; 44: 273-281.

21. KARP RJ, WILLIAMS C, GROUT J. Increased utilization of salty foods with age among pre-teenage black girls. J Nat Med Assoc 1980; 72: 197-200.

22. Loggie JMH, Horan mJ, Gruskin AB, Hohn AR, Dunbar JB, Havlik RJ. NHLbi, Workshop on Juvenile Hypertension. Proc Symp, Bethesda, Maryland, 26-27 May, 1983. 